\title{
Parto prematuro após uso de antimonial pentavalente: relato de um caso
}

\author{
Premature birth after the use of pentavalent antimonial: case report \\ Bruna Pinheiro Silveira², José Araújo Sobrinho', Lacínia Freire Leite², Maria das Neves \\ Andrade Sales', Maria do Socorro Araújo Gouveia', Renato Leal Mathias², \\ Ricardo Amorim Guedes Filho² e Sônia Maria Barbosa'
}

\begin{abstract}
Resumo Relata-se o caso de uma mulher de 19 anos, na $24^{a}$ semana de gravidez e com leishmaniose visceral. Tratada com antimonial pentavalente na posologia de $850 \mathrm{mg} /$ dia por 20 dias, ocorreu parto prematuro no quinto dia de tratamento e óbito da criança um dia após nascimento. Considerando a importância da protozoose no nosso meio e a raridade da associação com a gestação, julgamos de interesse a publicação do caso.
\end{abstract}

Palavras-chaves: Leishmaniose visceral. Gravidez. Antimonial pentavalente. Efeitos colaterais.

\begin{abstract}
A case is reported of a 19-year-old woman, at week 24 of gestation, with visceral leishmaniosis. She was treated with meglumine antimoniate at a dose of $850 \mathrm{mg} /$ day for 20 days. There occurred premature birth on day five of treatment and the neonate died one day after birth. Considering the importance of protozoiasis in our population and the rarity of the association with pregnancy, we resolved to publish the case.
\end{abstract}

Key-words: Visceral leishmaniasis. Pregnancy. Pentavalent antimonial. Side effect.

A leishmaniose visceral ou calazar concentra-se no Brasil, mais notadamente, na região nordeste, onde se verifica mais de $90 \%$ dos casos. Focos importantes também localizam-se nas demais regiões norte, sudeste e centro-oeste ${ }^{14}$.

Desde o ano de 1944, essa moléstia faz parte do quadro nosológico do Estado da Paraíba ${ }^{1}$. Evidências de urbanização da doença foram constatadas a partir de 1973 com a descrição de um foco no litoral paraibano ${ }^{5}$.

A possibilidade do envolvimento de mulheres em idade reprodutiva desperta preocupações quanto aos riscos de transmissão congênita e dos efeitos da medicação específica para a mãe e o concepto ${ }^{4}$.

\section{RELATO DO CASO}

L. B. C, 19 anos, sexo feminino, branca, casada, natural de Pombal, PB e procedente de Carfanaum, BA.

Gestante, no curso aproximado da $24^{\text {a }}$ semana de concepção, foi encaminhada para o Serviço de Doenças Infecciosas do Hospital Universitário Alcides Carneiro da Faculdade de Medicina de Campina Grande (PB) em outubro de 2000.

Relatava que há aproximadamente cinco meses apresentava febre diária, principalmente noturna, seguida de sudorese, que cedia com o uso de antitérmico (dipirona). Referia ainda anorexia, astenia, perda ponderal, escleras icterícas e colúria. Nos dois primeiros meses de evolução dos sintomas a paciente procurou atendimento médico, tendo sido levantada a suspeita de hepatite (SIC). Ocorreu um período de melhora, porém os sintomas retornaram.

No momento da internação, a paciente não apresentava outras queixas, afirmava precárias condições de vida e moradia e a existência de 3 casos de calazar na rua onde morava.

Ao exame físico encontrava-se com estado geral comprometido, afebril, normotensa, freqüência cardíaca de $72 \mathrm{bpm}$, palidez cutâneo-mucosa $(3+/ 4+)$ e icterícia nas escleróticas $(3+/ 4+)$. Foi evidenciado abdômen

\footnotetext{
1. Unidade de Infectologia do Departamento de Clínica Médica do Hospital Universitário Alcides Carneiro da Universidade Federal da Paraíba, Campina Grande, PB. 2. Hospital Universitário Alcides Carneiro.

Endereço para correspondência: Dra. Maria das Neves Andrade Sales. Rua Pedro II, 449, Prata, 58101-270 Campina Grande, PB.

e-mail: marianeves.andrade@bol.com.br

Recebido para publicação em 19/6/2001

Aceito em 10/6/2003
} 
gravídico com $20 \mathrm{~cm}$ de altura uterina, $86 \mathrm{~cm}$ de circunferência abdominal e esplenomegalia. Não foram observadas outras alterações.

Os exames complementares realizados no momento da internação mostraram: 1.900 .000 hemácias $/ \mathrm{mm}^{3}$. hemoglobina de $6.9 \mathrm{~g} / \mathrm{dl}$; hematócrito - 19,7\%; 1.260 leucócitos $/ \mathrm{mm}^{3}$ com desvio à esquerda e presença de granulações tóxicas nos neutrófilos; 126.000 plaquetas $/ \mathrm{mm}^{3}$. Proteinograma com globulinas de $3,9 \mathrm{~g} \%$ e albumina de $2,2 \mathrm{~g} \%$. Bilirrubina direta $-3,9 \mathrm{mg} / \mathrm{dl}$; Bilirrubina indireta - 1,93mg/dl; AST - 81u/l; ALT - 46u/l; fosfatase alcalina - 1037u/l; ferro sérico - $24 \mathrm{mg} / \mathrm{dl}$. O sumário de urina demonstrou bilirrubinúria. A ultra-sonografia evidenciou esplenomegalia e gestação única de 27 semanas. Os marcadores virais para hepatite B (ELISA anti HBs e $\mathrm{AgHBs}$ ) e $\mathrm{C}$ (ELISA anti-HCV) foram negativos. O exame de medula óssea mostrou presença de formas amastigotas de Leishmania chagasi.

Durante a internação, a paciente apresentou picos febris, epistaxe, hemoptise e equimoses disseminadas de grande diâmetro. Os batimentos e movimentos fetais estavam preservados e os exames laboratoriais mantiveram as mesmas anormalidades.
Decidiu-se iniciar o tratamento com uma cefalosporina de $3^{\mathrm{a}}$ geração (ceftazidima $1 \mathrm{~g}$ IV de 12/ 12hs) e logo após a confirmação do diagnóstico de Calazar, introduziu-se o antimonial pentavalente ( $\mathrm{N}$-metil glucamina $10 \mathrm{ml}+200 \mathrm{ml}$ de soro glicosado IV durante 1h uma vez ao dia).

A paciente entrou em trabalho de parto após o $5^{\circ}$ dia de uso do antimonial pentavalente, dando à luz, em outro serviço hospitalar, a um feto vivo, pré-termo, feminino (FVPTF). O recém-nascido foi assistido em unidade de terapia intensiva neonatal e apresentava ao exame físico: peso de $1.300 \mathrm{~kg}$, palidez acentuada, cianose, bradicardia, com crises de apnéia. Não foi evidenciado visceromegalias ou edemas. Faleceu no dia seguinte ao nascimento com diagnóstico de prematuridade, insuficiência respiratória aguda, doença da membrana hialina, acidose respiratória mista e interrogado calazar congênito.

Durante o tratamento, a paciente recebeu vários concentrados de hemácias, plaquetas e plasma. Recebeu alta no 22 dia de internação hospitalar após término de tratamento específico.

\section{DISCUSSÃO}

Tratava-se de uma jovem de 19 anos, no curso da segunda gestação, sem a realização de acompanhamento pré-natal sistemático embora tivesse recebido atendimento médico.

Suas manifestações clínicas prolongavam-se por cinco meses e apresentava uma hipótese diagnóstica prévia de hepatite.

A procedência do interior da Bahia e a referência de casos de leishmaniose visceral na rua onde residia, foram dados que mereceram considerações.

Comprovado o diagnóstico etiológico através do exame da medula óssea, a paciente e seus pais foram informados sobre as conseqüências possíveis da doença na gestação e dos efeitos colaterais das drogas indicadas. Houve concordância quanto ao tratamento.

A associação de leishmaniose visceral e gravidez não é freqüente ${ }^{24913}$, Thakur et $a^{12}$, em 938 casos, sendo 756 do sexo masculino e 182 femininos, encontraram a associação em apenas quatro deles.

O antimonial pentavalente é a droga de escolha para o tratamento da leishmaniose visceral, podendo ser administrada na grávida a partir do segundo trimestre na posologia de $850 \mathrm{mg} /$ dia por vinte dias ${ }^{13}$.

Outros autores ${ }^{210}$ contra indicam sua utilização na gestante. Um dos argumentos é a possibilidade de impregnação neural com o desenvolvimento de retardo mental.

Há quem coloque como droga ideal para essa fase a aminosidina na posologia de $12-16 \mathrm{mg} / \mathrm{kg} / \mathrm{dia}$ em aplicação intramuscular durante quinze a vinte dias ${ }^{2}$.

Caldas et $a^{\beta}$ relatam um caso da associação tratada com Anfotericina B (1 mg/kg/dia - 14 dias) com sucesso.

A toxicidade cardíaca é provavelmente a principal causa de morte em pacientes sob o regime terapêutico de drogas antimoniais ${ }^{6}$. Geralmente, os compostos trivalentes são mais tóxicos que os pentavalentes ${ }^{11}$.

No âmbito local já foi observado a refratariedade ao antimonial $\mathrm{N}$-metil glucamina em 3 crianças de um total de 80 estudadas ${ }^{10}$.

A informação sobre mutagenicidade, carcinogênese e teratogênese é escassa ${ }^{6}$.

Ocorrência de transmissão congênita tem sido aventada em número reduzido, até mesmo em mães que se mantiveram assintomáticas ${ }^{8}$.

No caso em estudo, não foi possível investigar a hipótese de transmissão congênita, em função da remoção da paciente para outras unidades hospitalares e por dificuldades técnicas.

\section{REFERÊNCIAS BIBLIOGRÁFICAS}

1. Almeida E. Um caso de leishmaniose visceral na Paraíba. Brasil Médico 59:82-83, 1944

2. Caldas AJMC, Aquino DMC, Costa JMC, Barral AP. Leishimaniose visceral na gravidez. In: Resumos do XXXVIII Congresso da
Sociedade Brasileira de Medicina Tropical, Foz do Iguaçu p. 315, 2002.

3. Fundação Nacional de Saúde. Controle, diagnóstico e tratamento da leishmaniose visceral. Normas técnicas, $1^{\mathrm{a}}$ edição, Ministério da Saúde, Brasília, 1994. 
4. Grandon L, Gaeta GB, Pellizzer G, Maisto A, Scalone A. Mediterranean visceral leishmanis in pregnancy. Scandinavia Journal Infectious Diseases 26:627-629, 1994.

5. Guedes GE. A leishmaniose visceral no litoral Paraibano. In: Resumos do XVI Congresso da Sociedade Brasileira de Medicina Tropical, Natal p. 452-458, 1980.

6. Léonard A, Gerber GB. Mutagenicity, carcinogenicity and teratogenicity of antimony compounds. Mutation Research 366:1-8, 1996.

7. Marzochi MCA, Marzochi KBF. Leishmanioses em áreas urbanas. Revista da Sociedade Brasileira de Medicina Tropical 30:162164, 1997.

8. Meinecken CK, Schottelius J, Oskam L, Fleischer B. Congenital transmission of asymptomatic mother to her child. Pediatrics 104:65, 1999.

9. Moraes C, Topyla VS, Paês RAP, Cuzy AF, Teleses JJA. Leishmaniose visceral durante a gestação. Revista Brasileira de Ginecologia e Obstetrícia 6:667-669, 1995.

10. Sobrinho JA, Rodrigues MLC, Gonzaga AAD, Celino ACBL. Aspectos epidemiológicos, laboratoriais e terapêuticos da leishmaniose visceral no hospital universitário Alcides Carneiro PB. Revista do Instituto Materno Infantil de Pernambuco 13:1316, 1999.

11. Tavares W. Antimoniais. In: Manual de antibióticos e quimioterápicos antiinfecciosos, $2^{\text {nd }}$ edição, Atheneu, Rio de Janeiro, p. 553-556, 1996.

12. Thakur CP, Sing RK, Hassan SM, Kumar R, Narain and Ashok Kumar S. Amphotericin B deoxycholate treatment of visceral leishmaniasis with never modes of administration and precautions: a study of 938 cases. Transactions of the Royal Society of Tropical Medicine and Hygiene 93:319-323, 1999.

13. Utilli R, Rambaldi A, Tripoli MF, Andreana A. Visceral leishmaniasis during pregnancy treated with meglumine antimoniate. Journal of Infectious Diseases-Clinical Study and Treatment 23:182-183, 1995.

14. Vieira JBF, Coelho GE. Leishmaniose visceral ou calazar; aspectos epidemiológicos e de controle. Revista da Sociedade Brasileira de Medicina Tropical 31:85-92, 1998. 